\title{
Influence of the CBA genetic background on sperm morphology and fertilization efficiency in mice with a partial Y chromosome deletion
}

\author{
J. Styrna ${ }^{1}$, W. Kilarski ${ }^{2}$ and H. Krzanowska ${ }^{1}$ \\ ${ }^{1}$ Department of Genetics and Evolution and ${ }^{2}$ Department of Cytology and Histology, \\ Institute of Zoology, Jagiellonian University, Ingardena 6, 30-060 Kraków, Poland
}

\begin{abstract}
Males of the B10.BR-Ydel mouse strain, with a deletion in the long arm of the $Y$ chromosome, were backcrossed to CBA females to introduce the Ydel chromosome to the genetic background of the CBA mice. The CBA-Ydel males (sixth backcross generation) had similar symptoms to those previously described for B10.BR-Ydel males (deterioration of sperm quality and of efficiency of fertilization), but these effects were much less pronounced, showing a favourable influence of the CBA genetic background. The CBA-Ydel males produced only $12 \%$ severely misshapen spermatozoa, and mating with B10.BR females gave $100 \%$ successful fertilization. Although nearly all sperm heads were abnormal ( $92 \%$ versus $6 \%$ in control males), most of the spermatozoa $(76 \%)$ had deformation only in the acrosomal part, that is, flat heads, which were not found in the control males. These abnormalities were analysed
\end{abstract}

in detail. As shown by differential staining, the acrosomes of the spermatozoa with flat heads were deformed; $18 \%$ of these acrosomes looked damaged, and often contained a vesicle, which stained in a similar way to the acrosome but lacked the reaction for acrosomal proteinase. Electron microscopy of testis sections revealed that deformations appeared in round spermatids as distortion of the acrosomal vesicle and asymmetrical position of the acrosomal granule; in many elongating spermatids the proximal end had a flat or concave shape, and the acrosomes contained a translucent vesicle. It is possible that the genes that are missing in the $\mathrm{Yq}$ deletion have some important regulatory function in the course of spermiogenesis, which may explain the various sperm defects observed in Y-del males.

\section{Introduction}

The $\mathrm{Y}$ chromosome plays an important role in reproduction of male mice, and deletions in this chromosome affect sperm morphology and function (for a review, see Burgoyne, 1998). Styrna et al. (1991a,b, 2002) showed that males from a mutant strain B10.BR-Ydel, with a deletion in the long arm of the $Y$ chromosome, have a high proportion $(88 \%)$ of spermatozoa with misshapen heads, as compared with males from the congenic B10.BR strain (31\%). The acrosomal region appears to be mostly affected; many sperm heads with flat acrosomes, occurring only in mutants (30\% of sperm population), are deficient in proteolytic enzymes, do not show a histochemical reaction for acrosine and, on electron micrographs, some spermatids lack acrosomal granules within the acrosomal vesicle.

The deletion in the $\mathrm{Y}$ chromosome affects not only the morphology of spermatozoa, but also their epididymal maturation and capacity to reach the ampulla and fertilize eggs. Small numbers of spermatozoa were

Email: styr@zuk.iz.uj.edu.pl recovered from the oviducts, and most matings with mutant males were sterile despite the presence of spermatozoa in the uterus. Even in the remaining matings, the rate of fertilization was lower than in control males (79\% versus 98\%; Styrna et al., 2002). Spermatozoa from B10.BR-Ydel males also had lower fertilization scores in vitro $(22 \%)$ than did spermatozoa from B10.BR males $(79 \%)$, which was mainly due to impaired capacitation (Xian et al., 1992).

The mutation in B10.BR-Ydel mice causes a deletion of more than two-thirds of the long arm of the $Y$ chromosome, as evidenced by a reduction in its size (Styrna et al., 1991b). It may be assumed that this $\mathrm{Yq}$ deletion caused a reduction in the number of copies of the candidate 'spermiogenesis gene' gene family, Ssty, mapping to this region (Burgoyne, 1998). There is evidence that abnormalities caused by the $\mathrm{Yq}$ deletion may be more severe in homozygous than in heterozygous mice, and are therefore dependent on the genetic background. For a $\mathrm{Yq}$ deletion in the $\mathrm{Y}$ chromosome derived from the RIII strain (RIII Yq-del), the frequency of sperm head abnormalities was higher when on an inbred C57BL/10 background (57\%), as compared 
with an MF1 random-bred background (7\%), although the proportional increase compared with controls was similar on the two backgrounds (Conway et al., 1994). It appears that the background of the B10.BR strain is even less adequate for sperm production, because as many as $20 \%$ of severely misshapen sperm heads are produced even in control males, and this may obscure the 'net' effect of the deletion. Therefore, to obtain a more specific picture of the defects caused by this deletion, the authors attempted to introduce the B10.BRYdel chromosome to the genetic background of the CBA strain, which is characterized by a very low proportion of sperm abnormalities (Krzanowska, 1969, 1981).

The aim of the present study was to compare the morphology and fertilizing capacity of spermatozoa of males carrying the B10.BR-Ydel chromosome on the CBA and B10.BR backgrounds. As it appeared that CBA-Ydel males produced as many as $76 \%$ of spermatozoa deformed in the acrosomal part of the head only (flat head), which appeared to be specific for this deletion, electron microscopy was used to study the details of this defect and its development during spermiogenesis.

\section{Materials and Methods}

\section{Animals}

The inbred strain of mice B10.BR-Ydel, with a partial deletion of the $\mathrm{Y}$ chromosome (Moriwaki et al., 1988), was obtained from The National Institute of Genetics (Mishima, Japan) in 1987, and then bred in the authors' department. B10.BR-Ydel males were mated with $\mathrm{CBA}$ females and the resulting males were backcrossed for six generations (B1-B6) to CBA females to introduce the deletion to the genetic background of the CBA/Kw strain (referred to as CBA). Males of the B4, B5 and B6 backcross generations (referred to as CBA-Ydel, or simply Ydel), and males born to B5 females mated with CBA males (control group with a normal $\mathrm{Y}$ chromosome), were used for experiments; in some cases CBA males were used too. Efficiency of fertilization was estimated in matings of Ydel and control males with females of the inbred B10.BR strain or with outbred mice maintained in the authors' department for nearly 40 years with maximal avoidance of inbreeding. All mice were given a commercial pelleted diet; the light was switched on between 09:00 $\mathrm{h}$ and 21:00 h. Male mice, aged 3-6 months, were caged singly for at least 3 days before being used in experiments. Animals were killed by cervical dislocation.

\section{Ethics of experimentation}

Experiments were performed in accordance with Polish legal requirements, under the licence of the Commission of Bioethics of the Jagiellonian University.

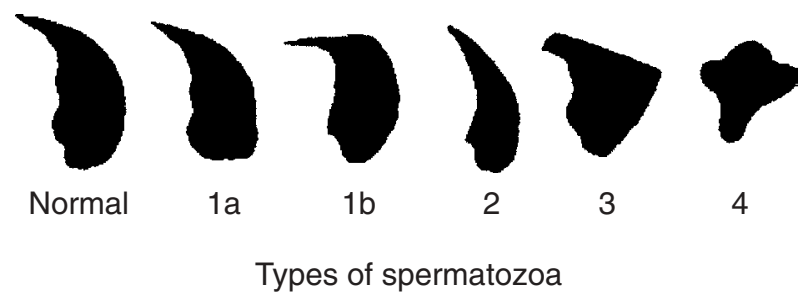

Fig. 1. Normal sperm head and different types of abnormality (reproduced from Styrna et al. (1991a), with some modifications).

\section{Testis mass and $X-Y$ chromosome dissociation}

After killing the male mice, the right testis of each mouse was weighed and processed for meiotic chromosome analysis according to the method reported by Meredith (1969). Slides were stained with $2 \%(\mathrm{w} / \mathrm{v})$ Giemsa in phosphate buffer, $\mathrm{pH}$ 6.8, for $30 \mathrm{~min}$. At least 50 spermatocytes at diakinesis or first meiotic metaphase were scored from each individual to estimate the percentage of dissociated $\mathrm{X}-\mathrm{Y}$ chromosomes and autosomes.

\section{Morphology of spermatozoa: light microscopy}

After pressing the cauda epididymides, the contents of the vasa deferentia were expressed into $0.2 \mathrm{ml}$ PBS at $\mathrm{pH} 7.2$ and allowed to disperse for a few minutes. A droplet of the sperm suspension was smeared on the slide, dried in air, fixed in a mixture of ethanol and acetic acid $(3: 1, v / v)$, air dried and stained with eosin $Y$ to assess the proportion of different types of sperm head abnormality (Fig. 1). Air-dried, unfixed smears were stained with a triple stain mixture (eosin $\mathrm{Y}$, fast green FCF and naphthol yellow S (Wako Pure Chemical Industries, Osaka) dissolved in 1.0\% (v/v) aqueous acetic acid) according to the technique described by Bryan (1970) to visualize the acrosomes. The method of Somfai et al. (2002) was applied to some sperm samples for simultaneous evaluation of sperm viability and acrosome integrity. In brief, a droplet of spermatozoa suspended in a physiological saline solution $(0.9 \%(\mathrm{w} / \mathrm{v}) \mathrm{NaCl})$ was mixed with a droplet of an aqueous solution of Trypan blue $(0.2 \% \mathrm{w} / \mathrm{v})$, smeared on the slide and air dried. After 2 days, the smears were fixed for 2 min in a mixture of $1 \mathrm{~mol} \mathrm{HCl} \mathrm{I}^{-1}(86 \mathrm{ml}), 37 \%(\mathrm{v} / \mathrm{v})$ formaldehyde $(14 \mathrm{ml})$ and neutral red $(0.2 \mathrm{~g})$, rinsed with tap and distilled water, stained for $24 \mathrm{~h}$ in $7.5 \%(\mathrm{v} / \mathrm{v})$ Giemsa solution (Sigma GS500; Sigma, St Louis, MO) in distilled water, differentiated in distilled water and air dried. The silver proteinate method of Yanagimachi and Teichman (1972) was used for cytochemical demonstration of acrosomal proteinase.

\section{Frequency of spermatozoa with a cytoplasmic droplet}

Small droplets of sperm suspension (obtained from the vasa deferentia, as described for examination of 
sperm morphology) were transferred to a slide, gently covered with a coverslip, and for each male 200 spermatozoa with progressive movements were inspected for the presence and localization of a cytoplasmic droplet.

\section{Water test}

The water test was performed as described by Fuse et al. (1993) for human spermatozoa: $10 \mu \mathrm{l}$ sperm suspension was mixed with $40 \mu \mathrm{l}$ distilled water on a clean glass slide. The mixture was covered with a coverslip and incubated for $5 \mathrm{~min}$ at $37^{\circ} \mathrm{C}$ before it was examined. The percentage of swollen spermatozoa (b-g type) was calculated after counting 200 spermatozoa from each male.

\section{Electron microscopy of spermatozoa and spermatids}

Ydel males aged 4-6 months (B4 and B5 generations) and control or CBA males were killed by cervical dislocation, and the whole testes and the cauda epididymides were immersed immediately in ice-cold fixative containing $2.5 \%(\mathrm{v} / \mathrm{v})$ glutaraldehyde, $2 \%(\mathrm{w} / \mathrm{v})$ paraformaldehyde (Fluka Chemie AG, Buchs) and 0.2\% $(\mathrm{w} / \mathrm{v})$ tannic acid in 0.1 mol cacodylate buffer $\mathrm{I}^{-1}$ ( $\mathrm{pH}$ 7.4) containing $10 \%$ sucrose. After 30 min of fixation, the tissues were fragmented into smaller pieces, fixed for several hours and stored overnight at $4{ }^{\circ} \mathrm{C}$. The tissues were rinsed in several changes of cacodylate buffer containing $10 \%(\mathrm{w} / \mathrm{v})$ sucrose and then again left overnight in the buffer at $4^{\circ} \mathrm{C}$. The small pieces of tissue were fragmented and post-fixed in $2 \%(\mathrm{w} / \mathrm{v})$ osmium tetroxide (Fluka Chemie) in cacodylate buffer ( $\mathrm{pH}$ 7.4) for $2 \mathrm{~h}$ at $4{ }^{\circ} \mathrm{C}$, washed briefly in distilled water and immersed in a saturated aqueous solution of uranyl acetate for $2 \mathrm{~h}$. After dehydration in a graded series of ethanol, the pieces of tissue were cleared in propylene oxide and embedded in Epon 812 epoxy resin. Semi-thin sections were cut on an LKB ultratome II with glass knives, stained in a mixture of toluidine blue and azure 2 in borax solution, and examined by light microscopy to select blocks in which the successive phases of spermiogenesis were observed in seminiferous tubules, and the mature spermatozoa in ductuli of epididymis, respectively. Ultrathin sections were cut with a diamond knife, mounted on 200-mesh copper grids and contrasted with uranyl acetate and lead citrate for $1 \mathrm{~min}$. The sections were examined and photographed in a JEOL 100 S-X transmission electron microscope at a constant magnification of $\times 5700$.

\section{Efficiency of fertilization and passage of abnormal spermatozoa into the oviducts}

Females were individually paired with Ydel or control males and killed on day 2 after the copulation plug was found. After flushing the oviducts with PBS, two types of ovum were recovered: fertilized (divided into two blastomeres with the second polar body) and unfertilized (not divided, without a polar body and containing a metaphase II spindle). For visualization of chromatin structure, the ova were dried in air on a microscope slide, fixed in a mixture of ethanol and acetic acid $(3: 1, v / v)$, dried again and stained with toluidine blue as described by Krzanowska and Lorenc (1983). Sporadically found fragmented ova, obviously from the previous ovulation, were not analysed.

Some females were killed at about 11:00 $\mathrm{h}$ on the day on which the copulation plug was found. Their oviducts were transferred to a drop of PBS containing $0.05 \%(\mathrm{w} / \mathrm{v})$ bovine testicular hyaluronidase (Laboratory of Sera and Vaccines, Warsaw) and ova were recovered by incision of the ampulla. After dispersion of granulosa cells, the ova were fixed and stained as described above. The oviducts were flushed with PBS with a pipette inserted into the cut end facing the uterus and the sample obtained was air dried. A droplet of the uterine contents was diluted with PBS and also air dried. Preparations of oviductal and uterine contents were fixed and stained with eosin Y, as described for examination of sperm morphology.

\section{Statistical analyses}

Unless stated otherwise, the differences between means were evaluated by one-way ANOVA. For statistical treatment, percentages were transformed to angles (Snedecor, 1955).

\section{Results}

The Ydel males had significantly larger testes and a lower proportion of spermatocytes with dissociated $\mathrm{X}-\mathrm{Y}$ chromosomes compared with control males (Table 1). The indices obtained for control males were similar to those found by Krzanowska (1989) for the inbred CBA males; the significance of the differences between them was not calculated because of the long time separating both investigations, with probable involvement of environmental dissimilarities.

\section{Sperm morphology: light microscope analysis}

The frequency of different types of sperm head abnormality (Fig. 1) was estimated on preparations stained with eosin Y. Ydel males had a very high proportion of abnormalities (92\% versus $6.5 \%$ in control males; Table 2 ), which was mainly due to type $1 \mathrm{~b}$ spermatozoa $(76 \%$ in Ydel males, but not found in control males), although most severely deformed heads (types 3 and 4) were also significantly more frequent in Ydel males. The indices of sperm abnormalities in control males were very similar to those of the inbred CBA males estimated 
Table 1. Characteristics of testes of Ydel, control and CBA male mice, aged 3-4 months

\begin{tabular}{|c|c|c|c|}
\hline Characteristic & $\begin{array}{c}\text { Ydel } \\
(n=10)\end{array}$ & $\begin{array}{l}\text { Control } \\
(n=10)\end{array}$ & $\begin{array}{c}\text { CBA } \\
(n=10)^{\mathrm{b}}\end{array}$ \\
\hline Body weight (g) & $33.9 \pm 1.61$ & $31.7 \pm 0.78$ & $25.0 \pm 0.47$ \\
\hline Testis mass (mg) & $82.3 \pm 2.99^{*}$ & $59.4 \pm 2.09$ & $50.8 \pm 0.88$ \\
\hline $\begin{array}{l}\text { Spermatocytes with } \\
\text { X-Y dissociated } \\
\text { chromosomes }^{\mathrm{a}}\end{array}$ & $28.7 \pm 2.25^{*}$ & $37.8 \pm 1.23$ & $32.3 \pm 2.32$ \\
\hline $\begin{array}{l}\text { Spermatocytes with } \\
\text { dissociated } \\
\text { autosomes }^{\mathrm{a}}\end{array}$ & $9.4 \pm 2.46$ & $11.6 \pm 1.86$ & $12.1 \pm 0.54$ \\
\hline
\end{tabular}

Values are mean \pm SEM.

a Percentages in angular transformation.

${ }^{b}$ From Krzanowska (1989).

*Significant difference compared with control males $(P<0.01)$.
Table 2. Proportion of spermatozoa with different types of abnormal head in the vas deferens of Ydel, control and CBA male mice

\begin{tabular}{|c|c|c|c|c|c|}
\hline \multirow{2}{*}{$\begin{array}{l}\text { Type of } \\
\text { sperm head } \\
\text { abnormality }\end{array}$} & \multicolumn{2}{|r|}{ Ydel $(n=7)$} & \multicolumn{2}{|c|}{ Control $(n=10)$} & \multirow{2}{*}{$\begin{array}{c}\mathrm{CBA}(n=6)^{\mathrm{a}} \\
\%\end{array}$} \\
\hline & $\%$ & Angle & $\%$ & Angle & \\
\hline $1 \mathrm{a}$ & 0.1 & $0.8 \pm 0.80$ & 0.4 & $2.6 \pm 1.21$ & 0.7 \\
\hline $1 b$ & 76.3 & $61.2 \pm 1.93^{* *}$ & 0 & 0 & 0 \\
\hline 2 & 3.3 & $10.0 \pm 0.70$ & 2.4 & $8.1 \pm 1.42$ & 1.3 \\
\hline 3 & 6.9 & $14.6 \pm 1.39 *$ & 2.4 & $8.0 \pm 1.59$ & 3.3 \\
\hline 4 & 5.5 & $12.7 \pm 1.44^{*}$ & 1.3 & $5.4 \pm 1.49$ & 1.2 \\
\hline Total & 92.2 & $75.2 \pm 2.52^{* *}$ & 6.5 & $14.4 \pm 1.53$ & 6.5 \\
\hline
\end{tabular}

Values are mean $(\%)$ or mean \pm SEM (angular transformation). aFrom Krzanowska (1981).

Significant difference compared with control males $(* * P<0.001$ $\left.{ }^{*} P<0.01\right)$.
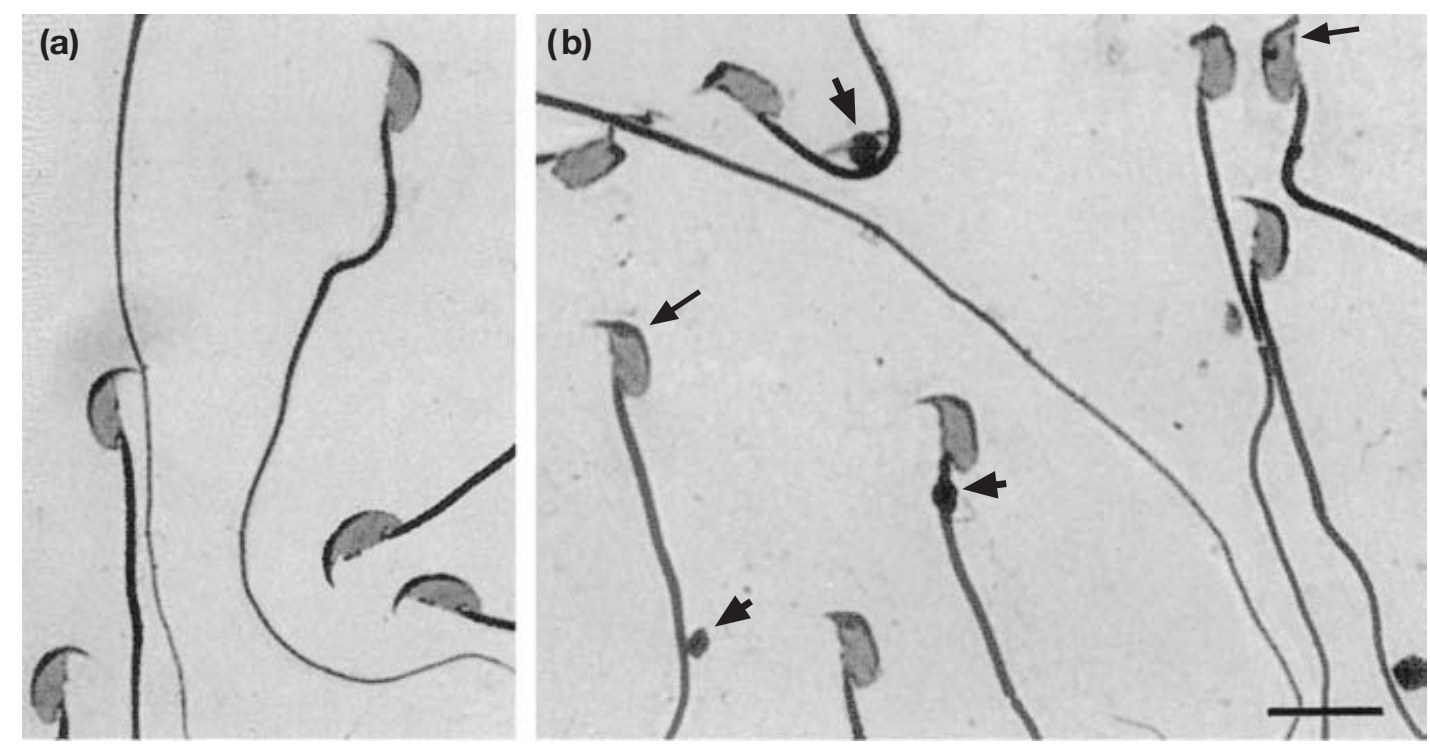

Fig. 2. Smears of spermatozoa from the vas deferens, stained by the method of Bryan (1970). (a) Typical sperm heads of a control male mouse with regularly shaped acrosomes, visible as dark lining of the convex contour of the head. (b) Flattened sperm heads of an Ydel male mouse with abnormally shaped acrosomes, some with a dark vesicle (arrows). Cytoplasmic droplets are indicated by arrowheads. Scale bar represents $10 \mu \mathrm{m}$.

by Krzanowska (1981) (Table 2). Type 1b spermatozoa had a flat anterior part of the head, but the intensity of this deformation was variable and difficult to classify; even heads classified as normal appeared to be slightly flattened when compared with spermatozoa of control males.

On preparations stained by the method of Bryan (1970), sperm heads of the control males had very regularly shaped acrosomes, in contrast to Ydel males, in which the acrosomes were irregularly flattened and often $(30 \%$ of spermatozoa) contained an intensively stained vesicle situated beneath the top or the convex surface of the acrosome (Fig. 2). Moreover, the distal parts of many acrosomes looked damaged; however, this could be due to the presence of dead spermatozoa. Therefore, acrosome integrity was evaluated on smears prepared by the method of Somfai et al. (2002), in which dead spermatozoa could be distinguished from live spermatozoa by the darker appearance of the postacrosomal part, stained with Trypan blue (Fig. 3). The acrosomes appeared purple after Giemsa staining; those of the Ydel males were flattened, irregular or damaged. The classification (Table 3) shows a significantly increased proportion of damaged acrosomes in live sperm heads of Ydel males (18\%) compared with control males $(0.6 \%)$. The proportion of live sperm heads without acrosomes and of dead spermatozoa was also increased in Ydel mice; however, these differences were not 
Table 3. Characteristics of spermatozoa from the vas deferens of Ydel and control male mice

\begin{tabular}{|c|c|c|c|c|c|c|}
\hline \multirow[b]{2}{*}{ Characteristics of spermatozoa } & \multicolumn{3}{|c|}{ Ydel } & \multicolumn{3}{|c|}{ Control } \\
\hline & $n$ & $\%$ & Angle (mean \pm SEM) & $n$ & $\%$ & Angle (mean \pm SEM) \\
\hline Live, with intact acrosomes ${ }^{\mathrm{a}}$ & 4 & 25.2 & $30.25 \pm 1.49^{* *}$ & 4 & 60.6 & $51.20 \pm 4.47$ \\
\hline Live, with damaged acrosomes ${ }^{\mathrm{a}}$ & 4 & 18.0 & $24.75 \pm 2.87^{* *}$ & 4 & 0.6 & $2.80 \pm 1.74$ \\
\hline Live, without acrosomes ${ }^{\mathrm{a}}$ & 4 & 4.7 & $11.50 \pm 2.63$ & 4 & 3.2 & $10.00 \pm 1.22$ \\
\hline Dead $^{\mathrm{a}}$ & 4 & 52.0 & $46.00 \pm 0.91$ & 4 & 35.6 & $36.40 \pm 4.13$ \\
\hline Without a cytoplasmic droplet & 10 & 46.2 & $42.60 \pm 3.10^{*}$ & 10 & 61.0 & $51.56 \pm 2.40$ \\
\hline Swollen in the water test & 9 & 28.8 & $32.11 \pm 2.10^{* *}$ & 9 & 42.4 & $40.71 \pm 1.76$ \\
\hline
\end{tabular}

aEstimated on preparations stained by the method of Somfai et al. (2002).

Significant difference compared with control males $\left({ }^{* *} P<0.01 ;{ }^{*} P<0.05\right)$.
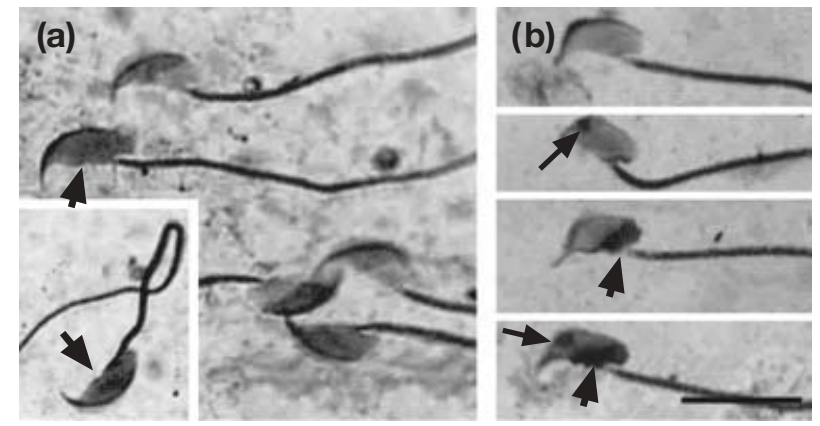

Fig. 3. Smears of spermatozoa from the vas deferens of mice, stained by the method of Somfai et al. (2002) to distinguish live spermatozoa from dead spermatozoa with darkly stained postacrosomal part (arrowheads). (a) Sperm heads of a control male with regular, intact acrosomes (inset: without acrosome). (b) Flattened sperm heads of a Ydel male; top to bottom: live with an acrosome, live without acrosome, dead with a damaged acrosome, dead without acrosome; dark vesicles indicated by arrows. Scale bar represents $10 \mu \mathrm{m}$.

significant. As in preparations stained by the method of Bryan (1970), about $30 \%$ of sperm heads, both with acrosomes or without acrosomes, contained a vesicle (Fig. 3b).

On smears prepared by the silver proteinate method of Yanagimachi and Teichman (1972), sperm heads of control males revealed proteinase activity as a precipitate of silver along the convex surface of the acrosomal caps (Fig. 4a,b). On sperm heads of Ydel males, an unstained 'vacuole' was visible under the precipitate (Fig. 4c). No differences in the frequency of spermatozoa without the acrosomes were noticed between smears from control and Ydel males.

Many spermatozoa expressed from the vasa deferentia contained a cytoplasmic droplet (Fig. 2b) situated at the end of the midpiece or, in rare cases $(4.0 \%$ and $1.8 \%$ in Ydel and control males, respectively; difference not significant), situated more proximally. The frequency of spermatozoa without a droplet was significantly higher in the vasa deferentia of the control
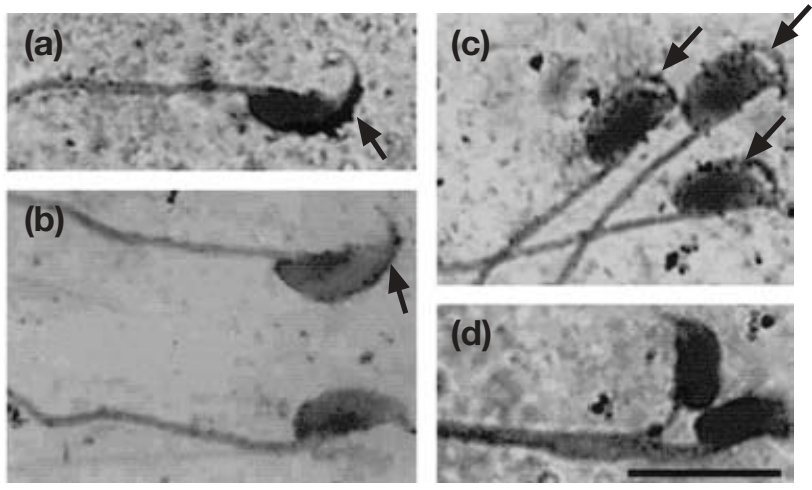

Fig. 4. Smears of spermatozoa from the vas deferens of mice, stained by the method of Yanagimachi and Teichman (1972) to demonstrate the distribution of acrosomal proteinase (arrows). (a,b) Sperm heads of a control male; acrosomal proteinase distributed along the convex contour of the head (on the bottom a head without acrosome). (c,d) Sperm heads of a Ydel male; (c) vacuoles (arrows) visible beneath the acrosomes; (d) without acrosomes. Scale bar represents $10 \mu \mathrm{m}$.

males than Ydel males (Table 3). In the water test, the frequency of swollen spermatozoa, which indicates cell integrity, was significantly higher in control males (Table 3).

\section{Electron microscopy}

Electron microscopy was used to investigate the developmental abnormalities in spermatozoa of Ydel mice and to determine the details of the structural defects of sperm heads that were observed using the light microscope. Spermatids from the testicular tubules and sperm heads from the cauda epididymides of Ydel (B4-B5 generations) and control (CBA and $\mathrm{B} 5 \times \mathrm{CBA})$ mice were studied.

In the control mice, most sperm heads in their mature form had semilunar shapes, with a remarkable perforatorium curved like a hook, characteristic for this species. The nuclei containing very condensed chromatin occupy all but the anterior tips of the heads. 

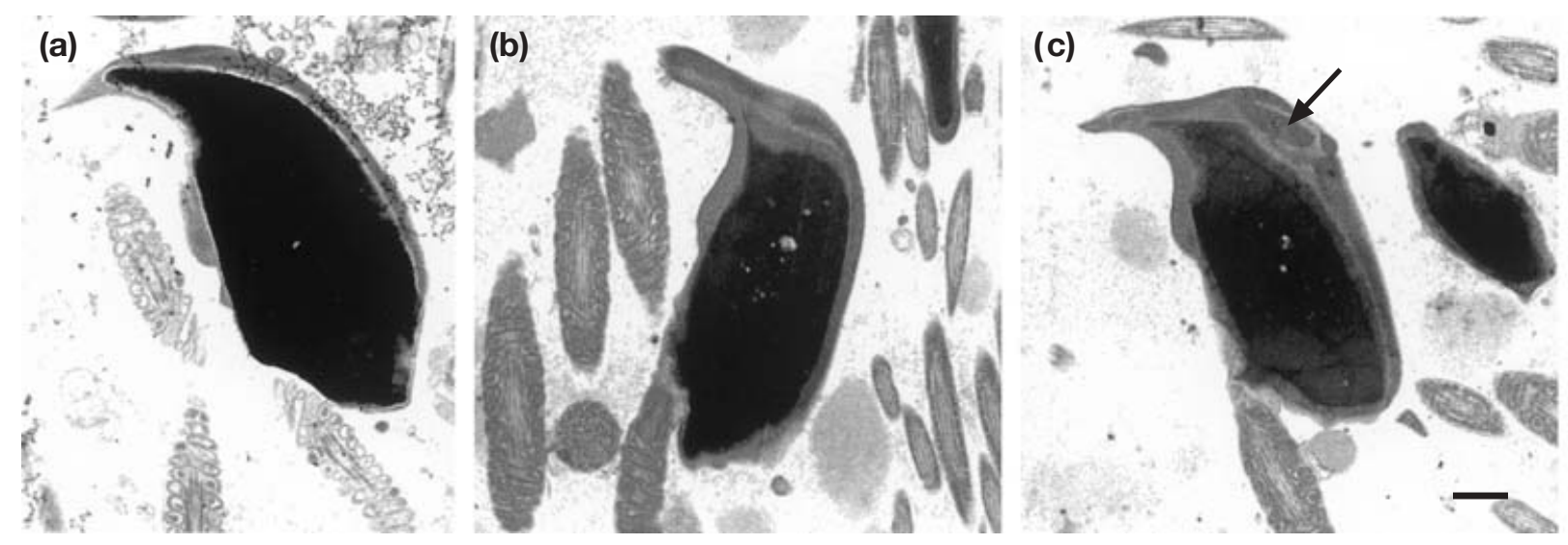

Fig. 5. Electron microscope sections of spermatozoa from the caput epididymidis of mice. (a) A typical sperm head from a control CBA male. $(b, c)$ Flattened sperm heads from a Ydel male, with deformed acrosomes; a vesicle is indicated by an arrow in (c). Scale bar represents $1 \mu \mathrm{m}$.

The heads are covered by acrosomes that spread out laterally to form a cap-like structure moulded on the posterior part of the heads. The convex surface of the acrosome in normal spermatozoa has an appearance of a regular crescent (Fig. 5a).

Mature spermatozoa of Ydel mice had aberrant heads that showed considerable diversity in their outlines. These abnormalities were observed particularly in the acrosomal region. The nuclei and the whole heads lacked the characteristic semilunar shape observed in spermatozoa of control mice. Instead, the anterior parts of the heads were abruptly bent and bluntly ended. The dorsal parts of the acrosomes and perforatoria followed the misshapen nuclei and were more or less perpendicularly oriented to the long axis of the sperm heads (Fig. 5b). In the favourable lateral sections through the sperm heads, granular inclusions accumulated in the form of an electron-dense material were observed in the dorsal part of the acrosomes. These pushed out the plasma membrane, giving the whole heads a humpbacked appearance (Fig. 5c).

Cross-sections of the testis tubules from Ydel and control mice were examined to elucidate the developmental steps leading to deformation of sperm heads. It should be noted that spermatogonia and spermatocytes did not show any detectable form of malformation. The first signs of defects appeared in the round spermatids at the capphase. In contrast to spermatids of control mice, with a regular, round nucleus covered in its anterior part with the acrosomal vesicle containing a centrally located acrosomal granule (Fig. 6a, arrow), many spermatids of Ydel mice displayed the following defects: (i) nuclear abnormalities, manifested by irregular thickening of the nuclear envelope that marks the polarity of the spermatid nucleus; in consequence the rounded curvature of the anterior poles of nuclei, observed in normal spermatids, was lost in Ydel spermatids (Fig. 6c, arrowhead); and (ii) deformations of the nuclei were accompanied by acrosomal defects, which included distortion of the
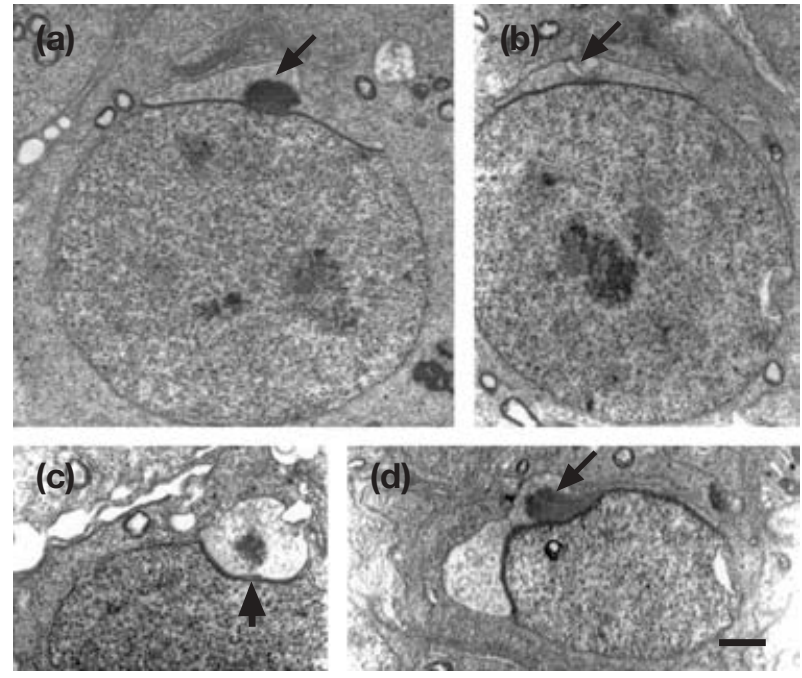

Fig. 6. Electron microscope sections of seminiferous tubules with round spermatids from mice. (a) Spermatid of a control CBA male, with an acrosomal vesicle containing a granule (arrow). (b-d) Abnormalities of spermatids of a Ydel male: (b) a vacuole (arrow) is present in the acrosomal vesicle instead of a granule; (c) acrosomal vesicle enlarged, with irregular thickening of the nuclear envelope (arrowhead); (d) acrosomal vesicle enlarged and irregular, with an asymmetrically situated granule (arrow). Scale bar represents $1 \mu \mathrm{m}$.

shape of the acrosomal vesicle, sometimes causing its invagination into the nucleus (Fig. 6c), and the displacement of the acrosomal granule to an asymmetric position (Fig. 6d). In some cases a 'vacuole' of low electron density was visible inside the acrosomal vesicle (Fig. 6b).

These abnormalities were more pronounced in the later phases of spermatid differentiation. The elongated spermatids of control mice, just before the onset of chromatin condensation, had a regular bullet-like assembly; their acrosomal caps were regularly contoured 

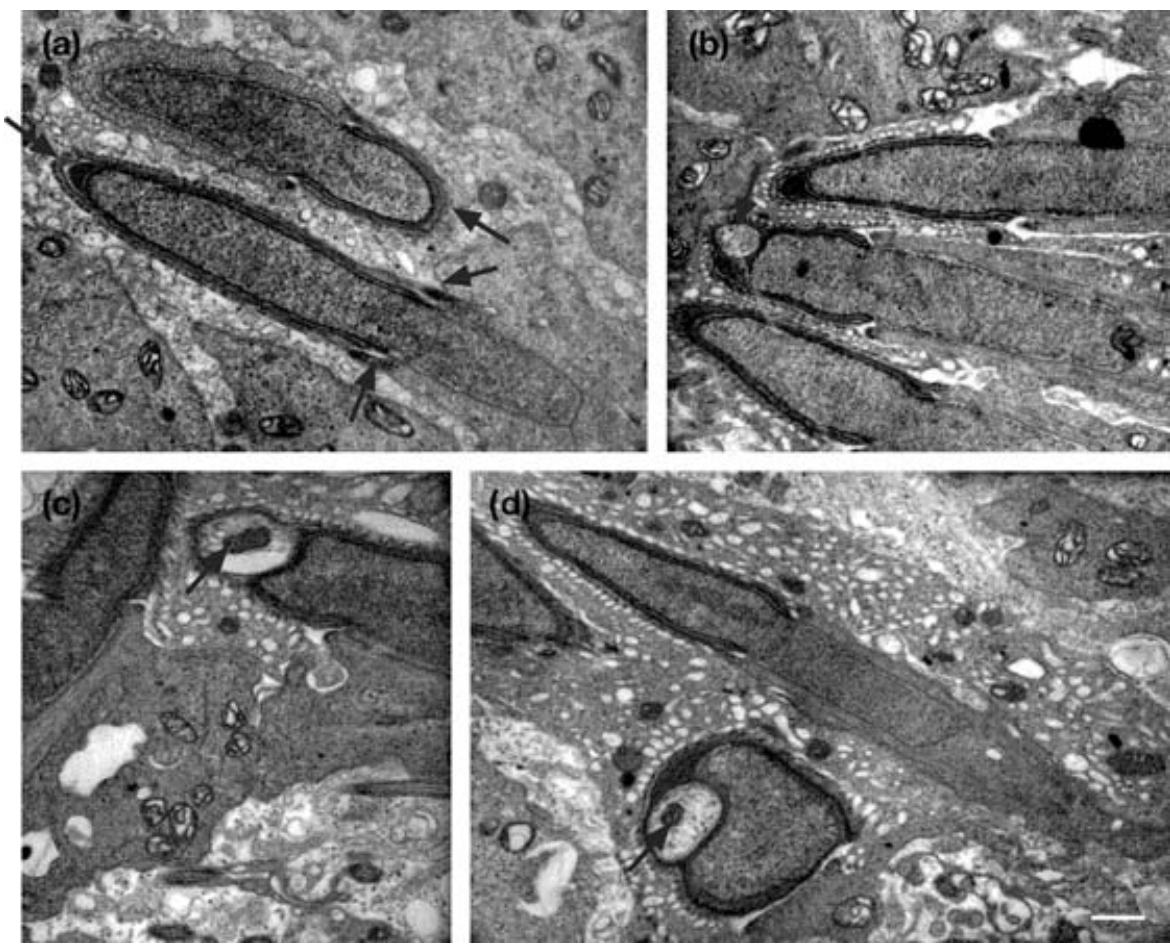

Fig. 7. Electron microscope sections of seminiferous tubules with elongating spermatids from mice. (a) Spermatids of a control CBA male with regular acrosomes (arrows). (b-d) Deformations of spermatids of a Ydel male: (b) flat sperm head with an acrosome containing a 'vacuole' (arrow); (c,d) deformed acrosomes with the translucent vesicle containing a centrally located granule (arrows). Scale bar represents $1 \mu \mathrm{m}$.

(Fig. 7a) and covered up by the layer of filaments that are part of the mantle derived from Sertoli cells. In many spermatids of Ydel males, at a similar stage of maturation, the proximal end was deformed, with a flat or even concave shape (Fig. $7 \mathrm{~b}-\mathrm{d}$ ), and equipped with deformed acrosomes containing translucent vesicles of an irregular form.

\section{Fertilization efficiency and passage of abnormal spermatozoa into the oviducts}

On day 2 after mating control or Ydel males with B10.BR females, $100 \%$ of fertilized ova were recovered (Table 4); in matings with outbred females the efficiency of fertilization was lower, but again the difference between the control and Ydel males was not significant. However, in a group of outbred females mated with Ydel males and killed on day 1 after mating, the percentages of fertilized ova were significantly lower than after mating with the control males, indicating a delay in fertilization.

The proportion of different types of abnormal spermatozoa recovered from the uterus of B10.BR females, on day 1 after mating with Ydel males, was the same as estimated for spermatozoa from the vasa deferentia (Fig. 8). In the oviductal flushings, only
Table 4. Efficiency of fertilization after mating B10.BR and outbred female mice with control or Ydel male mice

\begin{tabular}{|c|c|c|c|c|}
\hline \multirow{2}{*}{$\begin{array}{l}\text { Days after } \\
\text { copulation }\end{array}$} & \multirow[b]{2}{*}{ Strain of males } & \multicolumn{2}{|c|}{ Number of } & \multirow{2}{*}{$\begin{array}{l}\text { Proportion (\%) of } \\
\text { fertilized ova }\end{array}$} \\
\hline & & Males & Females & \\
\hline Second & Control & 4 & 6 (B10.BR) & $50 / 50(100.0)^{\mathrm{a}}$ \\
\hline Second & Ydel & 3 & 7 (B10.BR) & $52 / 52(100.0)^{\mathrm{a}}$ \\
\hline Second & Control & 5 & 7 (Outbred) & $43 / 47(91.5)^{a b}$ \\
\hline Second & Ydel & 5 & 7 (Outbred) & $48 / 58(82.6)^{b}$ \\
\hline First & Control & 5 & 5 (Outbred) & $51 / 66(77.3)^{b}$ \\
\hline First & Ydel & 3 & 5 (Outbred) & $8 / 48(16.7)^{\mathrm{c}}$ \\
\hline
\end{tabular}

${ }^{\mathrm{a}-\mathrm{c}}$ Differences between values marked by a different letter are significant $(P<0.01)$.

Chi-squared test of independence with Yates' correction.

the proportion of severely abnormal heads was lower: for type 3 abnormality, $1.2 \%$ in the oviduct versus $6.9 \%$ in the uterus, and for type $4,0.2 \%$ versus $5.9 \%$, respectively (after angular transformation of percentages, the differences were significant at $P<0.01$ ). For the group of outbred females it was not possible to perform such comparison, because of the small numbers of spermatozoa that could be recovered from their oviducts. 


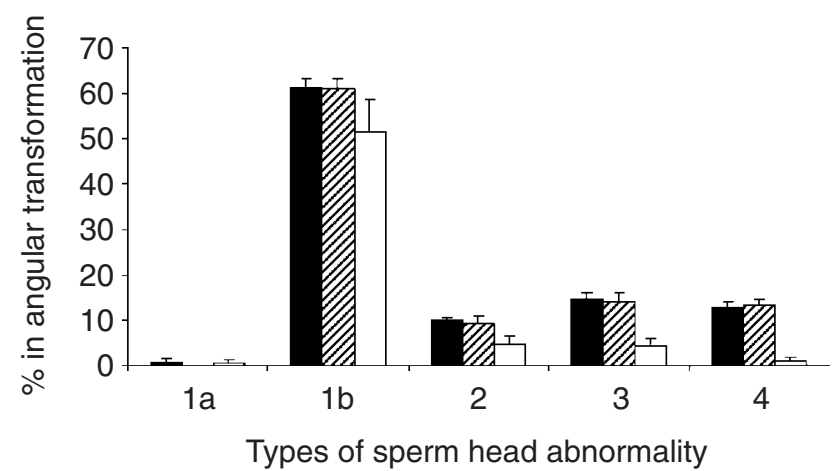

Fig. 8. Proportions (percentages in angular transformation; mean \pm SEM) of spermatozoa with different types of sperm head abnormality (Fig. 1) recovered from the vas deferens ( $\mathbf{\square})$ (Table 2), and from the uterus (四) and the oviducts $(\square)$ of B10.BR female mice mated with Ydel male mice.

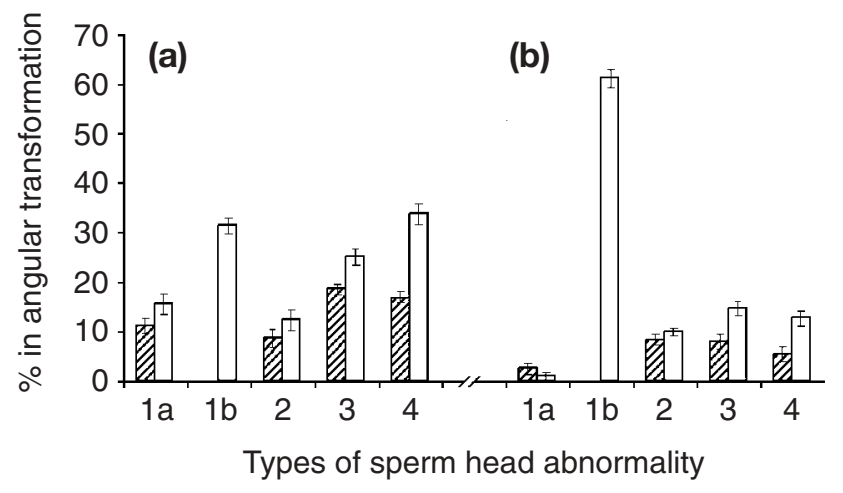

Fig. 9. Proportions (percentages in angular transformation; mean \pm SEM) of different types of sperm head abnormality in the vas deferens of control ( $\square)$ and Ydel ( $\square$ ) mice, on two genetic backgrounds: (a) B10.BR (Styrna et al., 2002) and (b) CBA (present study).

\section{Comparison of sperm morphology and fertilization capacity of males carrying the Ydel deletion on CBA and B10.BR backgrounds}

Males carrying the Ydel deletion had a high proportion of sperm head abnormalities compared with respective control males (Fig. 9), both on the B10.BR background (88\% versus $31 \%$; Styrna et al., 2002) and on the CBA background (92\% versus $6.5 \%$; present study; Table 2 ); in the case of the CBA background this was mainly due to the presence of rather slight, type $1 \mathrm{~b}$ abnormalities, amounting to $76 \%$. The proportional increase in the frequency of severe abnormalities (types 3 and 4) was higher in CBA-Ydel males $(12 \%$ versus $4 \%$ in control males) than in B10.BR-Ydel males (50\% versus $20 \%$ ), but the proportion of these abnormalities was much lower on the CBA background.

Higher sperm quality in CBA-Ydel compared with B10.BR-Ydel males was matched by their higher fertilization efficiency (Fig. 10), which was also influenced by the genotype of females; in the optimal combination (a)

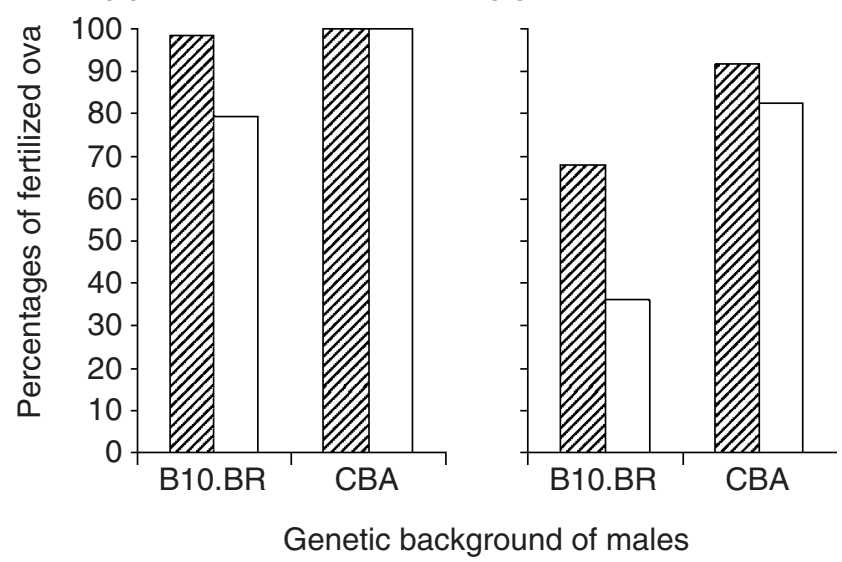

Fig. 10. Fertilization efficiency of control $(\square)$ and Ydel $(\square)$ males on two genetic backgrounds: B10.BR (Styrna et al., 2002; H. Krzanowska and J. Styrna, unpublished) and CBA (present study), depending on the strain of females ((a) B10.BR or (b) outbred).

(B10.BR females with CBA-Ydel males), 100\% fertilized ova were obtained, although the process of fertilization could be delayed (Table 4). Thus, the CBA genetic background exerted a favourable influence on both sperm morphology and the fertilizing capacity of males carrying the Ydel chromosome.

\section{Discussion}

After six generations of backcrossing Ydel males from the B10.BR strain to the CBA strain, most loci (theoretically 98\%; Falconer, 1960) should be homozygous for CBA alleles, but some may still be segregating. Therefore, the effect of different $\mathrm{Y}$ chromosomes on the same genetic background has been shown by making most comparisons between Ydel males (from $\mathrm{CBA} \times \mathrm{B} 5$ crosses; strain of female written first) and control males (from B5 $\times$ CBA crosses). The mice differed not only in the presence or absence of the deletion, but also in the non-recombining region of the $\mathrm{Y}$ chromosome, which was of B10.BR or CBA origin in the Ydel and control males, respectively. This difference could explain the lower testis mass observed in control compared with Ydel mice, because the $Y$ chromosome of the CBA strain has been shown to reduce testis size (Hayward and Shire, 1974; Shrenker and Maxson, 1986; Le Roy et al., 2001).

The proportion of sperm head abnormalities may be influenced by the genetic background, the source of the $Y$ chromosome and the effects of Ydel deletion, as well as interactions between these factors. As found by Krzanowska (1972), the $Y$ chromosome from the CBA strain improved sperm quality on the KE background, but, in the reciprocal situation, the $Y$ chromosome from the KE strain did not deteriorate sperm quality on the CBA 
background. This finding indicated that the composition of genes in the CBA strain may be so favourable for the production of morphologically normal spermatozoa that the difference in the $\mathrm{Y}$ chromosome failed to become manifest. If the same is the case with the B10.BR Y chromosome on the CBA background, it can be assumed that the comparison of sperm phenotypes in the Ydel and control males in the present study predominantly shows the effects of the deletion.

Sperm abnormalities of males carrying the Yq deletion have been found to be less severe on a heterozygous rather than on a homozygous background (Conway et al., 1994), which can be ascribed to the effects of heterosis. In the present study, the improvement in sperm morphology and fertilizing capacity occurred on the nearly homozygous background of the CBA strain, which is characterized by a very low proportion of sperm abnormalities (Krzanowska, 1969, 1981). Thus, not only heterotic effects but also a favourable combination of genes may reduce the effects of the $\mathrm{Yq}$ deletion, such that in favourable combinations of male and female genotypes, $100 \%$ fertilized ova may be achieved. The involvement of female genotype in fertilization efficiency was recently described for proacrosin-deficient mice (Nayernia et al., 2002).

The presence of flat sperm heads appears to be specific for the $\mathrm{Yq}$ deletion, as they were not observed in normal mice of various strains and crosses, but were found in all of the F1 hybrids investigated that were fathered by Ydel males (Styrna et al., 1991a) and in RIII Yq-del mice (Burgoyne, 1998; M. Szot, personal communication). On the CBA background, the frequency of this deformation was very high $(76 \%)$, and even sperm heads classified as normal (only $8 \%$ ) could also belong to this type. If so, practically all spermatozoa would be affected by this deformation, which, however, may be obscured if more severe abnormalities (types 2-4) are superimposed on it. Sperm head flattening is likely to be more conspicuous on the CBA background, which is characterized by spermatozoa with a large, very regular, semilunar head shape, than in B10.BR spermatozoa, which have shorter and less regular heads. As found by Styrna et al. (2002) for B10.BR-Ydel males, type $1 \mathrm{~b}$ spermatozoa produced by CBA-Ydel males were not prevented from passing to the oviducts and appear to take part in fertilization.

A characteristic feature of many flat sperm heads was the presence of a darkly stained vesicle which persisted even in sperm heads that had lost their acrosomes. Styrna et al. (1991b) noticed that many type $1 \mathrm{~b}$ sperm heads of B10.BR-Ydel males did not show a histochemical reaction for acrosomal proteinase, as evidenced by the method of Yanagimachi and Teichman (1972). In sperm heads of CBA-Ydel males, the acrosomal proteinase could be detected in type $1 \mathrm{~b}$ spermatozoa, but its distribution was distorted by the presence of a vesicle, which does not appear to contain acrosomal proteinase.
The occurrence of small, round vesicles lacking acrosin was recently described by Siruntawineti et al. (2002) in mice obtained from crosses of B10.BR-Ydel males with Acr-EGFP females, carrying a transgene with the protein-coding regions of pre-proacrosin and EGFP (green fluorescent protein) under the control of the acrosin gene promotor. Both epididymal spermatozoa and spermatids had green fluorescence in their acrosomes, but round small vesicles appearing in the acrosomes of elongating, step 9 spermatids were devoid of fluorescence, showing that they do not contain acrosin. The authors suggested that the vesicle may be eliminated from the acrosome of some elongating spermatids, but most of the vesicles remain and may correlate with the production of abnormal spermatozoa with flat heads.

Electron microscopy was used to study the development and details of flat sperm heads. As a result of the preponderance of type $1 \mathrm{~b}$ spermatozoa in Ydel mice in the present study, it can be assumed that the typical deformations found in the acrosomes of many elongating spermatids, and especially the presence of the vesicles, correspond to the vesicles described by Siruntawineti et al. (2002), and are involved in producing flat sperm heads. Deformations of the acrosome already occurred in round spermatids, which may not have been noticed in the investigations of Siruntawineti et al. (2002), who made their observations under the light microscope only. Both the shape of the nuclear envelope and the acrosome are involved in this abnormality but the underlying causes are not known.

Similar defects of acrosome formation were recently described by Martianov et al. (2002) in mice deficient in TLF (TBP-like factor), a protein that can act as a repressor or activator of RNA polymerase II transcription and is essential for spermatogenesis in mammals. In TLFdeficient mice the acrosomal granules did not coalesce in stage II-III spermatids, but were found in the cytoplasm in an asymmetric position, on the outer membrane of the nucleus or invaginated in the nucleus, and in the rare surviving elongating spermatids at stages IX$\mathrm{XI}$, the acrosomes were vacuolated or not properly associated with the head. The electron microscope pictures presented by the authors are very similar to those obtained in the present study; the phenotype of the spermatozoa is not known because of the arrest of spermatogenesis in TLF-deficient mice.

In normal spermiogenesis, many lysosomal proteins are removed from the acrosomal vesicle of the developing spermatids by degradation or other mechanisms (Ramalho-Santos et al., 2002). It is possible that disturbances of these mechanisms may lead to accumulation of components which failed to be removed, causing distortion of acrosome development. The fact that $\mathrm{Yq}$ deletion and TLF deficiency may lead to the development of similar sperm head abnormalities indicates that genes with important regulatory functions are probably 
responsible. The involvement of regulatory genes could also explain some other features of the spermatozoa from males carrying the $\mathrm{Yq}$ deletion: increase in frequency of severe sperm abnormalities, delayed maturation in the epididymis evidenced by the presence of a cytoplasmic droplet, lower indices of sperm integrity shown in water test and delayed fertilization. All these symptoms were found both on the B10.BR and on the CBA background (Styrna et al., 2002; and present study, respectively), but they were less pronounced on the CBA background.

The finding that the genetic background exerts an influence on the expression of a deletion in the $\mathrm{Y}$ chromosome may have a wider significance, particularly in relation to human fertility: owing to the high genetic heterogeneity of the human population, the same deletion may have more or less severe influence on fertility in different individuals, depending on the rest of the genotype. This could explain the recent observation that deletion of the $D A Z$ gene was associated with different phenotypic expression, including normal fertility (Calogero et al., 2002).

The authors are grateful to D. Lewandowska and P. Grzmil for help in performing the microphotography. The present study was partly supported by grants (DS./ZG/IZ/2003) from the Institute of Zoology of the Jagiellonian University.

\section{References}

Bryan JHD (1970) An eosin-fast green-naphthol yellow mixture for differential staining of cytologic components in mammalian spermatozoa Stain Technology 45 231-236

Burgoyne PS (1998) The role of Y-encoded genes in mammalian spermatogenesis Seminars in Cell and Developmental Biology 9 423432

Calogero AE, Garofalo MR, Barone N, Longo GA, De Palma A, Fichera M, Rappazzo G, D'Agata R and Vicari E (2002) Spontaneous transmission from a father to his son of a $Y$ chromosome microdeletion involving the deleted in a zoospermia (DAZ) gene Journal of Endocrinological Investigation 25 631-634

Conway SJ, Mahadevaiah SK, Darling SM, Capel B, Rattigan AM and Burgoyne PS (1994) Y353/B: a candidate multiple-copy spermiogenesis gene on the mouse Y chromosome Mammalian Genome 5 203-210

Falconer DS (1960) Introduction to Quantitative Genetics Oliver and Boyd, Edinburgh and London

Fuse H, Ohta S, Sakamoto M, Kazama T and Katayama T (1993) Hypoosmotic swelling test with a medium of distilled water Archives of Andrology 30 111-116

Hayward P and Shire JGM (1974) Y chromosome effect on adult testis size in the mouse (Mus musculus) Genetics 86 499-500

Krzanowska H (1969) Factor responsible for spermatozoon abnormality located on the $\mathrm{Y}$ chromosome in mice Genetical Research, Cambridge 13 17-24

Krzanowska H (1972) Influence of $Y$ chromosome on fertility in mice. In Proceedings of the International Symposium on Genetics of the Spermatozoon pp 370-386 Eds RA Beatty and S Gluecksohn-Waelsch. University of Edinburgh and Albert Einstein College of Medicine, Edinburgh and New York
Krzanowska H (1981) Sperm head abnormalities in relation to the age and strain of mice Journal of Reproduction and Fertility 62 385-392

Krzanowska H (1989) X-Y chromosome dissociation in mouse strains differing in efficiency of spermatogenesis: elevated frequency of univalents in pubertal males Gamete Research 23 357-365

Krzanowska H and Lorenc B (1983) Influence of egg investments on in vitro penetration of mouse eggs by misshapen spermatozoa Journal of Reproduction and Fertility 77 57-62

Le Roy I, Tordjman S, Migliore-Samour D, Degrelle H and Roubertoux PL (2001) Genetic architecture of testes and seminal vesicle weights in mice Genetics 158 333-340

Martianov I, Brancorsoni S, Gansmuller A, Parvinen M, Davidson I and Sassone-Corsi P (2002) Distinct functions of TBP and TLF/TRF2 during spermatogenesis: requirement of TLF for heterochromatic chromocenter formation in haploid round spermatids Development 129 945955

Meredith R (1969) A simple method for preparing meiotic chromosomes from mammalian testis Cytogenetics and Cell Genetics 26 254258

Moriwaki K, Suh D-S and Styrna J (1988) Genetic factors affecting sperm morphology in the mouse Mouse Newsletters 82138

Nayernia K, Adham IM, Shamsadin R, Müller C, Sancken U and Engel W (2002) Proacrosin-deficient mice and zona pellucida modifications in an experimental model of multifactorial infertility Molecular Human Reproduction 8 434-440

Ramalho-Santos J, Schatten G and Moreno D (2002) Control of membrane fusion during spermatogenesis and the acrosome reaction Biology of Reproduction 67 1043-1051

Shrenker P and Maxson C (1986) Effects of the DBA/1Bg Y chromosome on testis weight and aggression Behavior Genetics 16 263-270

Siruntawineti J, Yamagata K, Nakanishi T and Baba T (2002) Occurrence of small, round vesicles in the acrosome of elongating spermatids from a mouse mutant line with partial deletion of the $\mathrm{Y}$ chromosome Journal of Reproduction and Development 48 513-521

Snedecor GW (1955) Statistical Methods lowa College Press, Ames

Somfai T, Bodo Sz, Nagy Sz, Gócza E, Iváncsics J and Kovács A (2002) Simultaneous evaluation of viability and acrosome integrity of mouse spermatozoa using light microscopy Biotechnic and Histochemistry $\mathbf{7 7}$ $117-120$

Styrna J, Imai HT and Moriwaki K (1991a) An increased level of sperm abnormalities in mice with a partial deletion of the $\mathrm{Y}$ chromosome Genetical Research, Cambridge 57 195-199

Styrna J, Klag J and Moriwaki K (1991b) Influence of partial deletion of the $\mathrm{Y}$ chromosome on mouse sperm phenotype Journal of Reproduction and Fertility 92 187-195

Styrna J, Bilinska B and Krzanowska H (2002) The effect of a partial $Y$ chromosome deletion in B10.BR-Ydel mice on testis morphology, sperm quality and efficiency of fertilisation Reproduction, Fertility and Development 14 101-108

Xian M, Azuma S, Naito K, Kunieda T, Moriwaki K and Toyoda T (1992) Effect of a partial deletion of $\mathrm{Y}$ chromosome on in vitro fertilizing ability of mouse spermatozoa Biology of Reproduction 47 549553

Yanagimachi R and Teichman RJ (1972) Cytochemical demonstration of acrosomal proteinase in mammalian and avian spermatozoa by a silver proteinate method Biology of Reproduction 6 87-97

Received 7 April 2003.

First decision 1 July 2003.

Revised manuscript received 4 July 2003.

Accepted 4 July 2003. 